\title{
On the Inefficiency of Equilibria in Linear Bottleneck Congestion Games
}

\author{
Bart de Keijzer, Guido Schäfer, and Orestis A. Telelis ${ }^{\star}$ \\ Center for Mathematics and Computer Science (CWI) \\ Amsterdam, The Netherlands \\ \{B.de.Keijzer,G.Schaefer,0.Telelis\}@cwi.nl
}

\begin{abstract}
We study the inefficiency of equilibrium outcomes in bottleneck congestion games. These games model situations in which strategic players compete for a limited number of facilities. Each player allocates his weight to a (feasible) subset of the facilities with the goal to minimize the maximum (weight-dependent) latency that he experiences on any of these facilities. We derive upper and (asymptotically) matching lower bounds on the (strong) price of anarchy of linear bottleneck congestion games for a natural load balancing social cost objective (i.e., minimize the maximum latency of a facility). We restrict our studies to linear latency functions. Linear bottleneck congestion games still constitute a rich class of games and generalize, for example, load balancing games with identical or uniformly related machines with or without restricted assignments.
\end{abstract}

\section{Introduction}

Load balancing games constitute an important class of strategic games that capture many applications of practical relevance. These games model situations in which a set of strategically acting players (or jobs) compete for a limited number of resources (or machines). Every player chooses one of the resources available to him and assigns his weight (or load) to this resource. The latency of a resource depends on the total weight of the players using it. The goal of each player is to select a resource such that the latency that he experiences on this resource is minimized.

The study of load balancing games is motivated by the need for quantifying the inefficiency caused by selfish behavior of a set of autonomous players that utilize distributed processors upon which a system is built. The social cost objective of an assignment of loads to processors is measured by the makespan, i.e., the completion time of the most loaded machine, which reflects the distance from equi-distribution (balancing) of the load to the machines. Load balancing games have recently been studied extensively for a variety of different machine

\footnotetext{
* This work was carried out during the tenure of an ERCIM "Alain Bensoussan" Fellowship Programme.
} 
environments, including identical [15], uniformly related $[9,11,14,15]$, restricted assignment [5,11], and unrelated machines [2].

A natural extension of load balancing games are the bottleneck congestion games (BCGs) [6,12]. Here, every player chooses a subset of the resources (also called facilities in this context) from a set of feasible facility allocations and assigns his weight to each of these facilities. The goal of each player is to select a subset of the facilities such that the maximum latency over the chosen facilities is minimized. Bottleneck congestion games generalize, for example, load balancing games and network routing games, and have several applications in practice. Despite their importance, bottleneck congestion games have received only very little attention in the literature and are far from being well-understood. In this paper we study the inefficiency of stable outcomes in bottleneck congestion games.

Bottleneck congestion games essentially generalize the context of load balancing games by modeling the activity of each selfish player upon complexes of interrelated resources. This generalization brings the model closer to practice, as in most large scale computing systems the workload of a player occupies different components of the system simultaneously. For example, instantiations of such games emerge if the components form paths in networks, or if they correspond to parallel processors, etc. It is natural to assume that each player wants to balance his load across the different components available to him and hence attempts to minimize the maximum latency of a facility that he uses.

One of the most prominent solution concepts for the prediction of outcomes of rational behavior in strategic games is the Nash equilibrium concept. It describes outcomes that are resilient to unilateral player deviations. Throughout this paper we will focus exclusively on pure Nash equilibria. A more general solution concept is the strong equilibrium concept introduced by Aumann [3]. It describes outcomes of strategic games that are stable with respect to pure deviations of player subsets (also called coalitions). More precisely, an outcome of a strategic game is a strong equilibrium if no coalition of the players can deviate such that every member of the coalition strictly benefits. An outcome is said to be a $k$-strong equilibrium if this property holds for all coalitions of size at most $k$. Strong equilibria thus generalize the pure Nash equilibrium concept $(k=1)$. Very recently, Harks, Klimm and Möhring [12] showed that (under rather general assumptions) bottleneck congestion games always admit strong equilibria.

It is well known that equilibrium outcomes might be inefficient in the sense that they are suboptimal with respect to some socially desirable objective function. The price of anarchy (PoA) [15-17] has become the standard measure to assess the inefficiency of equilibrium outcomes. It is defined as the worst-case ratio (over all instances) of the maximum cost of a Nash equilibrium outcome and the cost of a socially optimal outcome. The strong price of anarchy (SPoA) and the $k$-strong price of anarchy ( $k$-SPoA) [2] refer to the natural adaptations of this measure to strong and $k$-strong equilibrium outcomes, respectively.

Contribution. We study the inefficiency of both pure Nash equilibria and strong equilibria of BCGs, under the natural assumption that the social cost of an outcome refers to the maximum latency of a facility. We restrict our studies to 


\begin{tabular}{|l||c|c||c|c|}
\hline \multicolumn{1}{|c||}{} & \multicolumn{2}{c||}{ id. facilities } & \multicolumn{2}{c|}{ arb. facilities (SPoA) } \\
\cline { 2 - 5 } & $k$-SPoA (lower) & SPoA & id. players & arb. players \\
\hline symmetric & $\max \left\{2,\left\lfloor\frac{m}{2 k}\right\rfloor+1\right\}$ & 2 & 2 & $O(m)$ \\
asymmetric & $\max \left\{\sqrt{2 m+\frac{1}{4}}-\frac{1}{2},\left\lceil\frac{m}{k-1}\right\rceil-1\right\}$ & $\Theta(\sqrt{m})$ & $O(\sqrt{n})$ & $\Theta(m)$ \\
\hline
\end{tabular}

Table 1: Summary of the bounds obtained for the SPoA and the $k$-SPoA of linear BCGs. The PoA of linear BCGs is at most $2 m-1$ and there is an asymptotically matching lower bound showing $\mathrm{SPoA} \geq m-1$.

linear bottleneck congestion games, where the latency of each facility is a linear function of the total weight assigned to it. These games still constitute a rich class of games and generalize, for example, load balancing games with identical or uniformly related machines with or without restricted assignments. We provide upper and lower bounds on the (strong) price of anarchy for symmetric and asymmetric linear BCGs (definitions will be given below). A summary of the results that we obtain in this paper is given in Table 1 . Here, we use $n$ and $m$ to refer to the number of players and facilities, respectively.

1. We show that both the PoA and the SPoA of linear BCGs is $\Theta(m)$. More precisely, we show that $m \leq \mathrm{PoA} \leq 2 m-1$ and $m-1 \leq \mathrm{SPoA} \leq m$.

2. We derive better bounds for identically weighted players. We prove that $\mathrm{SPoA}=2$ for symmetric linear BCGs and at most $O(\sqrt{n})$ and $O\left(\sqrt{m \gamma^{*}}\right)$ for asymmetric linear BCGs, where $\gamma^{*}$ refers to the cost of a socially optimal outcome.

3. We consider the case of identical facilities, i.e., all facilities have identical linear latency functions, and show that $\mathrm{SPoA}=\Theta(\sqrt{m})$.

4. We also give elaborate lower bounds on the $k$-SPoA for symmetric and asymmetric BCGs with identical facilities (see Table 1).

We remark that we also provide asymptotically tight worst-case examples for (directed) network congestion games (definitions will be given below).

Related Work. Network BCGs were considered first by Banner and Orda in [6]. The authors showed existence of pure Nash equilibria and provided an $\Theta(m)$ bound on the PoA for identical network links. Busch and Magdon-Ismail studied in [7] the PoA of network BCGs for identically weighted players. Very recently, Harks, Klimm and Möhring introduced general bottleneck congestion games and showed that strong equilibria are guaranteed to exist in these games.

As mentioned above, bottleneck congestion games generalize load balancing games, which have been studied intensively in recent years. Load balancing games were first studied by Koutsoupias and Papadimitriou [15]. Among other results, the authors provided a lower bound on the PoA of mixed Nash equilibria for the case of identical machines. Koutsoupias, Mavronicolas and Spirakis [14] and, independently, Czumaj and Vöcking [9], proved a matching upper bound. Czumaj and Vöcking also proved that PoA $=\Theta(\log m / \log \log m)$ for pure Nash equilibria. The same bound on the PoA was shown by Awerbuch et al. [5] for 
restricted assignments and identical machines. Gairing et al. [11] obtained independently the same bounds and proved $m-1 \leq \mathrm{PoA} \leq m$ for restricted assignments and uniformly related machines.

Andelman, Feldman and Mansour [2] were the first to study strong and $k$-strong equilibria in the context of load balancing games. They proved that $m \leq \mathrm{SPoA} \leq 2 m-1$ for the case of unrelated machines, which was tightened to exactly $m$ by Fiat et al. [10]. In this latter work it was also shown that the SPoA of strong equilibria for uniformly related machines is exactly $\Theta\left(\log m /(\log \log m)^{2}\right)$. For results in the context of more general scheduling games and associated scheduling policies (termed coordination mechanisms), the interested reader is referred to [13] and the references therein.

Bottleneck congestion games owe their name to their similarity to congestion games, which were introduced by Rosenthal [18]. In these games, the latency on each facility depends on the number of players using it (i.e., players have unit weights). The goal of each player is to minimize his cost which is defined as the sum (as opposed to the maximum for BCGs) of the latencies over the facilities used by the player. Rosenthal [18] proved the existence of pure Nash equilibria in congestion games. The price of anarchy of pure Nash equilibria for congestion games was resolved by Christodoulou and Koutsoupias [8] and, independently, by Awerbuch, Azar and Epstein [4]. It is shown in [8] that PoA = $\Theta(\sqrt{n})$ for asymmetric linear congestion games and the social cost being the maximum over the players' cost, and PoA $=\frac{5}{2}$ for (symmetric and asymmetric) linear congestion games and the social cost being the sum of the players' costs. Bounds for polynomial latency functions were also derived in [8]. Exact bounds for polynomial latencies and also for weighted players were developed in [1].

\section{Preliminaries}

In a bottleneck congestion game, we are given a set $N=[n]$ of $n$ players that want to utilize (non-cooperatively) a set $E=[m]$ of $m$ resources, which we also call facilities. ${ }^{1}$ Every player $i \in N$ has a positive weight (or load) $w_{i}>0$ and a strategy set $\Sigma_{i} \subseteq 2^{E}$ of feasible facility subsets from which he can choose. If player $i$ chooses facility subset $S_{i} \in \Sigma_{i}$, he allocates his entire weight $w_{i}$ to each facility $e \in S_{i}$. Let $\Sigma=\left(\Sigma_{1}, \ldots, \Sigma_{n}\right)$ be the set of all possible strategy choices of the players. A strategy profile $S=\left(S_{1}, \ldots, S_{n}\right) \in \Sigma$ specifies for each player $i \in N$ a strategy $S_{i} \in \Sigma_{i}$ that he has chosen. We define $N_{e}(S)$ as the set of players that have chosen facility $e \in E$ under $S$, i.e., $N_{e}(S)=\left\{i \in N \mid e \in S_{i}\right\}$. The total weight of facility $e \in E$ with respect to $S$ is defined as $w_{e}(S)=\sum_{i \in N_{e}(S)} w_{i}$.

Every facility $e \in E$ has a latency function $l_{e}: \Sigma \rightarrow \mathcal{R}^{+}$which satisfies the following three properties (see also [12]):

1. Non-negativity: $l_{e}(S) \geq 0$ for all $S \in \Sigma$.

2. Independence of irrelevant alternatives: $l_{e}(S)=l_{e}\left(S^{\prime}\right)$ for all $S, S^{\prime} \in \Sigma$ with $N_{e}(S)=N_{e}\left(S^{\prime}\right)$.

\footnotetext{
${ }^{1}$ We use notation $[k]$ to refer to the set $\{1, \ldots, k\}$ for some positive integer $k$.
} 
3. Monotonicity: $l_{e}(S) \geq l_{e}\left(S^{\prime}\right)$ for all $S, S^{\prime} \in \Sigma$ with $N_{e}(S) \supseteq N_{e}\left(S^{\prime}\right)$.

Given a strategy profile $S \in \Sigma$, every player $i \in N$ experiences an individual cost $c_{i}(S)$ equal to the latency of the most loaded facility that he uses, i.e., $c_{i}(S)=\max _{e \in S_{i}} l_{e}(S)$. We assume that every player $i \in N$ acts strategically and chooses his strategy $S_{i} \in \Sigma_{i}$ in order to minimize his own individual cost $c_{i}(S)$.

Aumann [3] introduced the notion of a strong equilibrium. Here we consider the refined notion of $k$-strong equilibrium. We use the standard notation $S_{-i}$ to refer to $\left(S_{1}, \ldots, S_{i-1}, S_{i+1}, \ldots, S_{n}\right)$. Similarly, we use $S_{I}$ and $S_{-I}$ to refer to the strategy profiles of $S$ induced by the players in $I$ and $N \backslash I$, respectively.

Definition 1. A strategy profile $S \in \Sigma$ is a $k$-strong equilibrium if for every non-empty player set $I \subseteq N$ with $|I| \leq k$ and every possible joint deviation $S_{I}^{\prime}$ of I there is at least one player $i \in I$ whose cost with respect to $S^{\prime}=\left(S_{-I}, S_{I}^{\prime}\right)$ is not better than with respect to $S$, i.e., $c_{i}\left(S_{-I}, S_{I}^{\prime}\right) \geq c_{i}(S)$.

With this definition, a strong equilibrium is a $k$-strong equilibrium with $k=$ $n$, and a pure Nash equilibrium is a $k$-strong equilibrium with $k=1$. Very recently, Harks, Klimm and Möhring [12] showed that strong equilibria always exist in BCGs satisfying Properties 1-3 above.

We are interested in characterizing the inefficiency of $k$-strong equilibria for BCGs. We assess the efficiency of a strategy profile $S$ by the maximum load of a facility under $S$. That is, the social cost $C(S)$ of a strategy profile $S \in \Sigma$ is defined as the maximum latency over all facilities, which is equivalent to the maximum cost over all players, i.e., $C(S)=\max _{e \in E} l_{e}(S)=\max _{i \in N} c_{i}(S)$. We will use $S^{*}$ to refer to an optimal strategy profile that minimizes $C(S)$ and denote its cost by $\gamma^{*}=C\left(S^{*}\right)$.

The $k$-strong price of anarchy $(k-S P o A)[2,15]$ refers to the worst-case ratio over all possible input instances of the maximum cost of a $k$-strong equilibrium and the cost $\gamma^{*}$ of the social optimum. We will simply refer to the price of anarchy $(P o A)$ and strong price of anarchy $(S P o A)$ for the 1-SPoA and the $n$ SPoA, respectively. One can easily make an example to show that the SPoA is unbounded in general. This motivates our studies of linear BCGs: We assume that the latency function $l_{e}$ of each facility $e \in E$ is a linear function of the total weight assigned to it, i.e., $l_{e}(S)=a_{e} w_{e}(S)$ for some $a_{e} \geq 0$. Linear BCGs constitute an important class of BCGs because they generalize, for example, various load balancing games as outlined in the Introduction.

A BCG is called a network $B C G$ if there exists a directed graph $G=(V, E)$ such that every player $i \in N$ is associated with a source $s_{i} \in V$ and a sink $t_{i} \in V$ and $i$ 's strategy set $\Sigma_{i}$ refers to the set of all directed paths from $s_{i}$ to $t_{i}$ in $G$. We call a game symmetric if all players have the same strategy set, i.e., $\Sigma_{i}=\Sigma_{j}$ for all $i, j \in N$; we call a game asymmetric otherwise. Observe that the above example corresponds to a network BCG, but is not symmetric.

Unless stated otherwise, we assume subsequently that all player weights are at least one, i.e., $w_{i} \geq 1$ for every $i \in N$, and that the coefficient of each latency function is at least one, i.e., $l_{e}(S)=a_{e} w_{e}(S)$ with $a_{e} \geq 1$ for every $e \in E$. These 
assumptions are without loss of generality as we can always enforce them by scaling the weights and coefficients appropriately.

\section{$3 \quad$ Arbitrary Facilities}

In this section, we derive bounds on the PoA and SPoA of linear BCGs. We consider both the general and the identical player case.

\subsection{Arbitrary Players}

We first consider the most general case of arbitrary linear latency functions and arbitrary player weights. We show that the PoA is at most $2 m-1$ in this case. We obtain a better bound of $m$ on the SPoA and present an almost tight lower bound.

Theorem 1. The price of anarchy of linear BCGs is at most $2 m-1$ and at least $m$.

Proof. Let $S$ be a pure Nash equilibrium with $\operatorname{cost} C(S)=\alpha \gamma^{*}$ for some $\alpha \geq 1$. We prove by induction that for every integer $k, 1 \leq k<\frac{\alpha+1}{2}+1$, there is a set $E_{k}$ of $k$ distinct facilities such that for every $e \in E_{k}, l_{e}(S) \geq(\alpha-k+1) \gamma^{*}$.

The claim holds true for $k=1$ because there must exist a facility $e \in E$ with latency $l_{e}(S)=\alpha \gamma^{*}$. Suppose that the induction hypothesis holds true for $k<\frac{\alpha+1}{2}$. We will prove that there exists a set $E_{k+1}$ of $k+1$ distinct facilities such that $l_{e}(S) \geq(\alpha-k) \gamma^{*}$ for every $e \in E_{k+1}$. Choose from $E_{k}$ a facility $\hat{e}$ with smallest $a_{e}$, i.e., $\hat{e}=\arg \min _{e \in E_{k}} a_{e}$. By the induction hypothesis, we have $l_{\hat{e}}(S) \geq(\alpha-k+1) \gamma^{*}>k \gamma^{*}$. Let $I_{\hat{e}}=N_{\hat{e}}(S)$ be the set of players choosing $\hat{e}$ under $S$. Note that $w_{\hat{e}}(S) \geq l_{\hat{e}}(S) / a_{\hat{e}}>k \gamma^{*} / a_{\hat{e}}$. Consider the strategies that the players in $I_{\hat{e}}$ choose under $S^{*}$ and suppose for the sake of a contradiction that for every $i \in I_{\hat{e}}, S_{i}^{*} \cap E_{k} \neq \emptyset$. Then there is a facility $e \in E_{k}$ with $w_{e}\left(S^{*}\right) \geq$ $w_{\hat{e}}(S) / k>\gamma^{*} / a_{\hat{e}}$. By the choice of $\hat{e}$, we have $l_{e}\left(S^{*}\right)=a_{e} w_{e}\left(S^{*}\right)>\gamma^{*}$, which is a contradiction to the definition of $\gamma^{*}$. Thus there is a player $j \in I_{\hat{e}}$ that chooses a strategy $S_{j}^{*}$ that is disjoint from $E_{k}$. Note that for every $e \in S_{j}^{*}$ we have $a_{e} w_{j} \leq \gamma^{*}$. Since $S$ is a pure Nash equilibrium, player $j$ cannot decrease his cost by deviating to $S_{j}^{*}$ and thus there is some facility $e^{\prime} \in S_{j}^{*}$ such that:

$$
l_{e^{\prime}}(S)=\left(a_{e^{\prime}} w_{e^{\prime}}(S)+a_{e^{\prime}} w_{j}\right)-a_{e^{\prime}} w_{j} \geq c_{i}(S)-a_{e^{\prime}} w_{j} \geq l_{\hat{e}}(S)-\gamma^{*} \geq(\alpha-k) \gamma^{*}
$$

The inductive step follows by setting $E_{k+1}=E_{k} \cup\left\{e^{\prime}\right\}$. By choosing $k=$ $\left\lceil\frac{\alpha+1}{2}\right\rceil<\frac{\alpha+1}{2}+1$, we obtain that there is a set $E_{k} \subseteq E$ with $\left|E_{k}\right| \geq k$ and thus $m \geq\left|E_{k}\right| \geq k \geq \frac{\alpha+1}{2}$. We conclude that $\mathrm{PoA}=\alpha \leq 2 m-1$.

The following instance shows that $\mathrm{PoA} \geq m$, even for symmetric BCGs with identical facilities and identical players. Consider a BCG with player set $N=[n]$ and facility set $E=[m]$ with $m=n$. Every player $i \in N$ has unit weight $w_{i}=1$ and the latency function $l_{e}(S)$ of every $e \in E$ is the identity function, 
i.e., $l_{e}(S)=w_{e}(S)$. Suppose that each player $i \in N$ has strategy set $\Sigma_{i}=2^{E}$. If every player chooses a distinct facility we obtain an optimal strategy profile $S^{*}$ with $\gamma^{*}=1$. On the other hand, consider the strategy profile $S$ in which every player allocates all facilities in $E$. This is a pure Nash equilibrium of cost $C(S)=m$.

We derive a better upper bound on the SPoA for linear BCGs. The following key lemma will be used several times in the paper.

Lemma 1. Let $S$ be a strong equilibrium and let $I_{\lambda} \subseteq I$ be a non-empty subset of the players such that for every $i \in I_{\lambda}$ we have $c_{i}(S) \geq \lambda \gamma^{*}$, for some $\lambda \geq 1$.

1. Then there is a player $i \in I_{\lambda}$ and a facility $e \in S_{i}^{*}$ such that $l_{e}\left(S_{-I_{\lambda}}\right) \geq$ $(\lambda-1) \gamma^{*}$.

2. Suppose that $I_{\lambda}$ is maximal. Then there is a player set $T_{\lambda} \subseteq N \backslash I_{\lambda}$ with $w\left(T_{\lambda}\right) \geq \lambda-1$ and for every $i \in T_{\lambda}$ we have $(\lambda-1) \gamma^{*} \leq c_{i}(S)<\lambda \gamma^{*}$.

Proof. We first prove the first part of the lemma. Note that for every player $i \in I_{\lambda}$ and every $e \in S_{i}^{*}$ we have

$$
l_{e}\left(S_{I_{\lambda}}^{*}\right) \leq l_{e}\left(S^{*}\right) \leq \gamma^{*} .
$$

Suppose for the sake of a contradiction that for every player $i \in I_{\lambda}$ and for every $e \in S_{i}^{*}$ it holds that $l_{e}\left(S_{-I_{\lambda}}\right)<(\lambda-1) \gamma^{*}$. Consider the strategy profile $S^{\prime}=\left(S_{-I_{\lambda}}, S_{I_{\lambda}}^{*}\right)$ in which the players in $I_{\lambda}$ deviate to their optimal strategies in $S^{*}$. Using (1), we obtain for every $i \in I_{\lambda}$ and for every $e \in S_{i}^{*}$ :

$$
l_{e}\left(S^{\prime}\right)=l_{e}\left(S_{I_{\lambda}}^{*}\right)+l_{e}\left(S_{-I_{\lambda}}\right)<\gamma^{*}+(\lambda-1) \gamma^{*}=\lambda \gamma^{*} .
$$

Thus, for every $i \in I_{\lambda}, c_{i}\left(S^{\prime}\right)=\max _{e \in S_{i}^{*}} l_{e}\left(S^{\prime}\right)<\lambda \gamma^{*}$, which is a contradiction to $S$ being a strong equilibrium.

We next prove the second part of the lemma. Let $i \in I_{\lambda}$ be a player and $e \in S_{i}^{*}$ be a facility satisfying $l_{e}\left(S_{-I_{\lambda}}\right) \geq(\lambda-1) \gamma^{*}$. Define $T_{\lambda}$ as the set of players that choose $e$ under $S$ but are not contained in $I_{\lambda}$, i.e., $T_{\lambda}=N_{e}(S) \backslash I_{\lambda} \subseteq N \backslash I_{\lambda}$. We have

$$
a_{e} w\left(T_{\lambda}\right)=l_{e}\left(S_{T_{\lambda}}\right)=l_{e}\left(S_{-I_{\lambda}}\right) \geq(\lambda-1) \gamma^{*} .
$$

Since $e \in S_{i}^{*}$ and $w_{i} \geq 1$ for every $i \in N$, we have $a_{e} \leq \gamma^{*}$. Thus, $w\left(T_{\lambda}\right) \geq$ $\lambda-1$. Consider an arbitrary player $i \in T_{\lambda}$. By the above we have, $c_{i}(S) \geq l_{e}(S) \geq$ $l_{e}\left(S_{T_{\lambda}}\right) \geq(\lambda-1) \gamma^{*}$. Moreover, by the maximality of $I_{\lambda}$ and since $i \notin I_{\lambda}$, we have $c_{i}(S)<\lambda \gamma^{*}$.

Remark 1. Observe that in the above proof we exploit the linearity of the latency functions only in (2). In fact, we can draw exactly the same conclusion if all latency functions are sub-additive, i.e., for every $e \in E, l_{e}(x+y) \leq l_{e}(x)+l_{e}(y)$ for every $x, y \in \mathcal{R}^{+}$. As a consequence, all our upper bounds on the SPoA (which exploit Lemma 1) hold for sub-additive latency functions. 
Theorem 2. The strong price of anarchy of linear BCGs is at most $m$.

Proof. Let $S$ be a strong equilibrium with cost $C(S)=\alpha \gamma^{*}$ for some $\alpha>1$. For an arbitrary real value $1<\lambda \leq \alpha$, let $I_{\lambda}$ be the maximal non-empty set of players $I_{\lambda}=\left\{i \in N \mid c_{i}(S) \geq \lambda \gamma^{*}\right\}$. Applying Lemma 1, we obtain a player set $T_{\lambda}$ such that for every $i \in T_{\lambda}$ we have $(\lambda-1) \gamma^{*} \leq c_{i}(S)<\lambda \gamma^{*}$. Moreover, $w\left(T_{\lambda}\right) \geq \lambda-1>0$ because $\lambda>1$ and thus $T_{\lambda}$ is non-empty. We can thus identify a family $F=\left\{T_{\alpha}, T_{\alpha-1}, \ldots, T_{\alpha-k}\right\}$ of $k+1$ player sets that are non-empty and pairwise disjoint, where $k$ is the largest integer satisfying $\alpha-k>1$. Every set $T_{\lambda} \in F$ identifies at least one distinct facility $e \in E$ with $(\lambda-1) \gamma^{*} \leq l_{e}(S)<\lambda \gamma^{*}$. Moreover, there is one facility $e \in E$ with $l_{e}(S)=\alpha \gamma^{*}$. We conclude that $m \geq|F|+1=k+2 \geq \alpha$ and thus SPoA $=\alpha \leq m$.

Theorem 3. The strong price of anarchy is at least $m-1$ in general linear $B C G$ s and at least $\frac{m+1}{3}$ in single-sink linear network BCGs.

The proof of this result is deferred to the full version. The lower bound of $m-1$ can also be derived by a construction in [11].

\subsection{Identical Players}

We next derive an upper bound on the SPoA for linear BCGs if the weights of all players are identical. In this subsection, we assume without loss of generality that the weight of each player $i \in N$ is $w_{i}=1$.

Theorem 4. The strong price of anarchy is at most $O\left(\min \left\{\sqrt{n}, \sqrt{m \gamma^{*}}\right\}\right)$ for linear BCGs with identical players and 2 for linear symmetric BCGs with identical players.

Proof. We prove the first part of the theorem. Let $S$ be a strong equilibrium with cost $C(S)=\alpha \gamma^{*}$ for some $\alpha>1$. As in the proof of Theorem 2, we can apply Lemma 1 to identify a family $F=\left\{T_{\alpha}, T_{\alpha-1}, \ldots, T_{\alpha-k}\right\}$ of $k+1$ player sets that are non-empty and pairwise disjoint, where $k$ is the largest integer satisfying $\alpha-k>1$. Each such set $T_{\lambda} \in F$ contains at least $\lambda-1$ players, i.e., $\left|T_{\lambda}\right| \geq\lceil\lambda-1\rceil$ for every $\alpha-k \leq \lambda \leq \alpha$. Moreover, there is at least one player that experiences a congestion of $\alpha \gamma^{*}$. Thus

$$
n \geq 1+\sum_{\lambda=1}^{\lceil\alpha-1\rceil} \lambda \geq 1+\frac{\alpha(\alpha-1)}{2} .
$$

Solving for $\alpha$ we obtain $\alpha \leq \frac{1}{2}+\sqrt{2 n-3 / 2}$. Recall that we assume without loss of generality that $a_{e} \geq 1$ for every $e \in E$ and thus $\gamma^{*} \geq n / m$. We therefore also obtain $\alpha \leq \frac{1}{2}+\sqrt{m \gamma^{*}-3 / 2}$. Thus SPoA $\leq \alpha=O\left(\min \left\{\sqrt{n}, \sqrt{m \gamma^{*}}\right\}\right)$.

We next prove the second part of the theorem. In a strong equilibrium $S$, at least one player $i \in N$ must have $\operatorname{cost} c_{i}(s) \leq \gamma^{*}$ since otherwise the grand coalition could deviate to the socially optimal strategy profile. Suppose there is a player $j \in N$ whose cost is more than two times larger than the cost of $i$. 
Consider the deviation $S^{\prime}=\left(S_{-j}, S_{i}\right)$ where player $j$ deviates to the strategy of player $i$. Then $c_{j}\left(S^{\prime}\right) \leq \max _{e \in S_{i}} a_{e}\left(w_{e}(S)+1\right) \leq \max _{e \in S_{i}} 2 a_{e} w_{e}(S) \leq 2 c_{i}(S)$, which is a contradiction to $S$ being a strong equilibrium.

The following example establishes the tightness of this bound: Let $N=[3]$ and $E=[6]$. The strategy set of every player is $\left\{\sigma_{1}=\{1\}, \sigma_{2}=\{2,3\}, \sigma_{3}=\right.$ $\left.\{4,5\}, \sigma_{4}=\{2,5,6\}\right\}$. The social optimum is $S_{i}^{*}=\sigma_{i}$ for every player $i \in[3]$ with $\gamma^{*}=1$. A strong equilibirum is given by $S_{1}=\sigma_{4}$ and $S_{2}=S_{3}=\sigma_{1}$. The cost of $S$ is $C(S)=2$. It is easy to see that this example is a network BCG .

\section{Identical Facilities}

In this section, we study the SPoA for the case of linear BCGs with identical facilities, i.e., the latency function of every facility $e \in E$ is $l_{e}(S)=w_{e}(S)$.

Theorem 5. The strong price of anarchy of linear BCGs with identical facilities is at most $-\frac{1}{2}+\sqrt{2 m+\frac{1}{4}}$ in general and exactly 2 in case of symmetric games.

Proof. For the symmetric case we claim that in any stronf equilibrium configuration $S$, there is at least one player $i_{0}$ with $c_{i_{0}}(S) \leq \gamma^{*}$. Indeed, if $c_{i}(S)>\gamma^{*}$ for all players, then the grand coalition would deviate to $S^{*}$. Now for any player $i$ we have $\gamma^{*} \geq w_{i}$. Let $i$ be any player with $e \in S_{i}$ such that $c_{i}(S)=l_{e}(S)=C(S)$. Consider unilateral deviation $S_{i}^{\prime}=S_{i_{0}}$ of $i$. Then, because $S$ is also a pure Nash equilibrium, $C(S)=c_{i}(S) \leq c_{i_{0}}(S)+w_{i} \leq 2 \gamma^{*}$. A tight lower bound has already been presented in Theorem 4 .

For the asymmetric case let the cost of a strong equilibrium $S$ be $C(S)=\alpha \gamma^{*}$, for some $\alpha>1$. Similar to the proof of Theorem 2, let $I_{\lambda}$ be the maximal non-empty set of players $I_{\lambda}=\left\{i \in N \mid c_{i}(S) \geq \lambda \gamma^{*}\right\}$ for some $1<\lambda \leq \alpha$. By Lemma 1 , we obtain a player set $T_{\lambda}$ such that for every $i \in T_{\lambda}$ we have $(\lambda-1) \gamma^{*} \leq c_{i}(S)<\lambda \gamma^{*}$. We can refine the argument given in the proof of Lemma 1 to bound the weight of $T_{\lambda}$ for identical facilities as follows: By inequality (3), we have $w\left(T_{\lambda}\right) \geq(\lambda-1) \gamma^{*} / a_{e}=(\lambda-1) \gamma^{*}$, where the last equality holds because for identical facilities $a_{e}=1$ for every $e \in E$. Moreover, $w\left(T_{\lambda}\right) \geq(\lambda-1) \gamma^{*}>0$ because $\lambda>1$ and thus $T_{\lambda}$ is non-empty. That is, we can identify a family $F=\left\{T_{\alpha}, T_{\alpha-1}, \ldots, T_{\alpha-k}\right\}$ of $k+1$ player sets that are non-empty and pairwise disjoint, where $k$ is the largest integer satisfying $\alpha-k>1$. Moreover, by construction we have $I_{\alpha} \cap T_{\lambda}=\emptyset$ for every $T_{\lambda} \in F$ and $w\left(I_{\alpha}\right) \geq \alpha \gamma^{*}$ since facilities are identical. The total weight $w(N)$ is then:

$$
w(N) \geq \alpha \gamma^{*}+\sum_{\lambda=\alpha-k}^{\alpha} w\left(T_{\lambda}\right) \geq \alpha \gamma^{*}+\sum_{\lambda=\alpha-k}^{\alpha}(\lambda-1) \gamma^{*} \geq \alpha \gamma^{*}+\sum_{\lambda=0}^{\alpha-1} \lambda \gamma^{*}
$$

The latter equals $\frac{1}{2} \alpha \gamma^{*}(1+\alpha)$. Observe that $\gamma^{*} \geq w(N) / m$ because facilities are identical. We obtain $2 m \geq \alpha(1+\alpha)$ or equivalently $\alpha \leq-\frac{1}{2}+\sqrt{2 m+1 / 4}$. Since $\mathrm{SPoA} \leq \alpha$ the claim follows. 
Theorem 6. The strong price of anarchy of linear BCGs with identical players and identical facilities is at least $-\frac{1}{2}+\sqrt{2 m+\frac{1}{4}}$ in general and at least $-\frac{1}{4}+$ $\frac{1}{2} \sqrt{2+2 m}$ in single-sink network BCGs .

Proof. We give a family of instances with $m$ facilities and $n=\Theta(m)$ unweighted players, which we turn into a family of network instances subsequently. Consider a partition of the set of players $N$ into $q$ subsets, $N=\bigcup_{j=1}^{q} P_{j}$, where $\left|P_{j}\right|=j$, $j \in[q]$. Denote players in $P_{j}$ by $p_{j i}, i \in[j]$. For each subset $P_{j}$ make a new set of $j$ distinct facilities $E_{j}=\left\{e_{1}^{j}, \ldots, e_{j}^{j}\right\}$. Define $E_{q+1}=E_{1}$. For every player $p_{j i} \in P_{j}, i \in[j]$, set the strategy space of $p_{j i}$ to:

$$
\Sigma_{p_{j i}}=\left\{\{e\} \mid e \in E_{j}\right\} \cup\left\{E_{j+1}\right\}
$$

For the socially optimal configuration set $S_{p_{j i}}^{*}=\left\{e_{i}^{j}\right\}$. Then $C\left(s^{*}\right)=1$. Now consider the configuration $S$ where $S_{p_{j i}}=E_{j+1}$ for $i \in[j], j \in[q]$. The cost of $S$ is defined by the latency of the unique facility $e=e_{1}^{1} \in E_{1}$ and is $C(S)=l_{e}(S)=\left|P_{q}\right|=q$. For every player $p \in P_{j}$, we have $c_{p}(S)=j$. We claim that $S$ is a strong equilibrium. Consider any deviation of any coalition $I \subseteq N$. Denote by $S_{p}^{\prime}$ the novel strategy that any player $p \in I$ adopts and let $S^{\prime}$ denote the resulting configuration. Notice that for the unique player $p \in P_{1}$ we have $c_{p}(S)=1$, hence no deviation may lessen his cost and $P_{1} \cap I=\emptyset$.

Let $j=\min \left\{j^{\prime} \mid P_{j^{\prime}} \cap I \neq \emptyset\right\}$; then $j \geq 2$, and $S_{j}^{\prime} \cap E_{j} \neq \emptyset$. For all $j-1$ players $p_{j-1, i} \in P_{j-1}$ it holds that $S_{p_{j-1, i}}=E_{j}$, because $I \cap P_{j-1}=\emptyset$. Hence, $c_{j}\left(S^{\prime}\right)=j-1+1=j=c_{j}(S)$. In any deviation of any coalition $I$, at least one player does not have incentive to deviate jointly with $I$ and hence $\mathrm{SPoA} \geq q$. Now for $q$ we have $m=\left|\cup_{j} E_{j}\right|=\sum_{j=1}^{q} j=\frac{q(q+1)}{2}$, which yields $q \geq-\frac{1}{2}+\sqrt{2 m+1 / 4}$.

We convert the example into a network BCG . To grant access to players in $P_{j-1}$ to facilities in $E_{j}$, we make a path of length $3,\left\{\left(s_{j}, u_{j i}\right),\left(u_{j i}, v_{j i}\right),\left(v_{j i}, t\right)\right\}$, for every facility $e_{i}^{j} \in E_{j}, i \leq j-1$ and a length-2 path $\left\{\left(s_{j}, u_{j j}\right),\left(u_{j j}, t\right)\right\}$ for $e_{j}^{j}$. Let $A_{j}$ be the set of arcs in these paths. Node $s_{j}$ is the source of all players in $P_{j}$ and $t$ is a common sink for all players. Now we add auxiliary arcs $A_{j}^{\prime}=\left\{\left(v_{j i}, u_{j, i+1}\right) \mid i \in[j-1]\right\}$. And, finally, an arc $\left(s_{j-1}, u_{j 1}\right), j \in\{2, \ldots, q\}$, by which players $P_{j-1}$ gain access to $A_{j}$. For the last group of players we add an $\operatorname{arc}\left(s_{q}, t\right)$. Let us illustrate the analog of configuration $S$ on the constructed network. All players in $p_{j i} \in P_{j}, i \in[j]$, play the same path strategy:

$$
\begin{aligned}
S_{j i} & =\left\{\left(s_{j}, u_{j+1,1}\right\}\right. \\
& \cup\left\{\left(u_{j+1, r}, v_{j+1, r}\right),\left(v_{j+1, r}, u_{j+1, r+1}\right) \mid r \in[j-1]\right\} \\
& \cup\left\{\left(u_{j+1, j}, v_{j+1, j}\right),\left(v_{j+1, j}, t\right)\right\}
\end{aligned}
$$

and $S_{i q}=\left(s_{q}, t\right)$ for $i \in[q]$. See Fig. 1a for an example with $q=4$. The proof that $S$ is strong is analogous to the proof given for the non-network example. For the optimal configuration we set $S_{j i}^{*}=\left\{\left(s_{j}, u_{j i}\right),\left(u_{j i}, v_{j i}\right),\left(v_{j i}, t\right)\right\}$, for each player $p_{i j} \in P_{j}, i<j$, and $S_{j j}=\left\{\left(s_{j}, u_{j j}\right),\left(u_{j j}, t\right)\right\}$. The number of links $m$ is: 


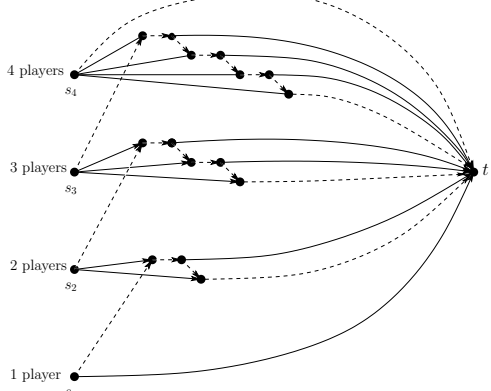

(a) SPoA on identical links.



(b) 2-SPoA for 6 identical players on identical links; player indices mark links used by each player.

Fig. 1: Lower bound constructions for Strong and 2-Strong Equilibria on identical links.

$$
m=\sum_{j=1}^{q}\left(\left|A_{j}\right|+\left|A_{j}^{\prime}\right|\right)+q=\sum_{j=1}^{q}(3 j-1+(j-1))+q-1=2 q^{2}+q-1
$$

which yields $q \geq-\frac{1}{4}+\frac{1}{2} \sqrt{2+2 m}$.

\subsection{Lower Bounds On $k$-Strong Equilibria}

For the $k$-SPoA of symmetric and general BCGs with identical facilities we show:

Theorem 7. The k-strong price of anarchy of linear BCGs is at least:

1. $\left\lfloor\frac{m}{2 k}\right\rfloor+1$ for symmetric BCGs and $\left\lceil\frac{m+2}{6 k}\right\rceil$ for symmetric network BCGs, when $2 \leq k \leq \frac{m}{2}$.

2. $\left\lceil\frac{m}{k-1}\right\rceil-1$ in general, when $2 \leq k \leq \frac{3}{4}+\frac{1}{2} \sqrt{\frac{1}{4}+2 m}$.

The proofs of these results are deferred to the full version. Figure $1 \mathrm{~b}$ presents a 2 -strong equilibrium for 6 identical players and 34 identical links. The maximum latency over all links under this configuration is 3 . The social optimum has cost 1 and emerges when all players use link-disjoint paths to reach $t$ from $s$.

\section{References}

1. Aland, S., Dumrauf, D., Gairing, M., Monien, B., Schoppmann, F.: Exact Price of Anarchy for Polynomial Congestion Games. In: Proceedings of the 23rd Annual Symposium on Theoretical Aspects of Computer Science (STACS), LNCS 3884. pp. 218-229. Springer (2006) 
2. Andelman, N., Feldman, M., Mansour, Y.: Strong price of anarchy. In: Proceedings of the 18th ACM-SIAM Symposium on Discrete Algorithms (SODA). pp. 189-198. ACM Press (2007)

3. Aumann, R.J.: Acceptable points in games of perfect information. Pacific Journal of Mathematics 10, 381-417 (1960)

4. Awerbuch, B., Azar, Y., Epstein, A.: Large the price of routing unsplittable flow. In: Proceedings of the 37th ACM Symposium on Theory of Computing (STOC). pp. 57-66 (2005)

5. Awerbuch, B., Azar, Y., Richter, Y., Tsur, D.: Tradeoffs in worst-case equilibria. Theoretical Computer Science 361(2-3), 200-209 (2006)

6. Banner, R., Orda, A.: Bottleneck Routing Games in Communication Networks. IEEE Journal on Selected Areas in Communication 25(6), 1173-1179 (2007)

7. Busch, C., Magdon-Ismail, M.: Atomic Routing Games on Maximum Congestion. Theoretical Computer Science 410(36), 3337-3347 (2009)

8. Christodoulou, G., Koutsoupias, E.: The price of anarchy of finite congestion games. In: Proceedings of the 37th ACM Symposium on Theory of Computing (STOC). pp. 67-73. ACM Press (2005)

9. Czumaj, A., Vöcking, B.: Tight bounds for worst-case equilibria. ACM Transactions on Algorithms 3(1) (2007)

10. Fiat, A., Kaplan, H., Levy, M., Olonetsky, S.: Strong Price of Anarchy for Machine Load Balancing. In: Proceedings of the 34th International Colloquium on Automata, Languages and Programming (ICALP), LNCS 4596. pp. 583-594. Springer (2007)

11. Gairing, M., Lücking, T., Mavronicolas, M., Monien, B.: The price of Anarchy for Restricted Parallel Links. Parallel Processing Letters 16(1), 117-132 (2006)

12. Harks, T., Klimm, M., Möhring, R.: Strong nash equilibria in games with the lexicographical improvement property. In: Proceedings of the 5th Workshop on Internet and Network Economics (WINE), LNCS 5929. pp. 463-470. Springer (2009)

13. Immorlica, N., Li, E., Mirrokni, V.S., Schulz, A.S.: Coordination mechanisms for selfish scheduling. Theoretical Computer Science 410(17), 1589-1598 (2009)

14. Koutsoupias, E., Mavronicolas, M., Spirakis, P.G.: Approximate Equilibria and Ball Fusion. Theory of Computing Systems 36(6), 683-693 (2003)

15. Koutsoupias, E., Papadimitriou, C.H.: Worst-case Equilibria. In: Proceedings of the 16th Annual Symposium on Theoretical Aspects of Computer Science (STACS), Springer LNCS 1563. pp. 404-413 (1999)

16. Koutsoupias, E., Papadimitriou, C.H.: Worst-case equilibria. Computer Science Review 3(2), 65-69 (2009)

17. Papadimitriou, C.H.: Algorithms, Games, and the Internet. In: Proceedings of the 33rd ACM Symposium on Theory of Computing (STOC). pp. 749-753. ACM Press (2001)

18. Rosenthal, R.W.: A class of games possessing pure-strategy nash equilibria. International Journal of Game Theory 2(1), 65-67 (1973) 